\title{
PROTOTIPAGEM E A MULTIDISCIPLINARIDADE DA INOVAÇÃO: EM BUSCA DE UMA CARACTERIZAÇÃO HOLÍSTICA
}

Ivan Santiago Braga (ivansantiagobraga@ @otmail.com) - Universidade Federal de Minas Gerais

Raoni Barros Bagno (rbagno@ dep.ufmg.br) - Universidade Federal de Minas Gerais

\section{RESUMO}

A prototipagem é um conceito recorrente na Gestão de Desenvolvimento de Produtos (GDP), tendo fortes implicações na dinâmica de desenvolvimento de inovações em suas esferas técnica e gerencial. Nas últimas décadas, ideias fundamentais associáveis ao conceito transbordaram para vários outros contextos de estudo em que a inovação é protagonista das discussões, como o Design de Serviços, as Tecnologias de Manufatura Rápida, a Engenharia de Software, o Design Thinking, o Movimento "Maker" e o Empreendedorismo de Base Tecnológica. Atualmente, tais incursões proporcionam diferentes entendimentos e ênfases, mas falta um esforço de análise transversal que reintegre o conceito a partir das nuances de cada área. O presente trabalho tem como objetivo revisitar a discussão sobre prototipagem na GDP, identificar diferentes áreas que estudam ou utilizam o conceito e realizar uma análise comparativa do entendimento que essas possuem. Para isso, adota-se como metodologia uma revisão bibliográfica sistemática, complementada por entrevistas semiestruturadas com especialistas das disciplinas envolvidas. Como resultados, têm-se um compêndio de disciplinas que adotam o conceito e o mapeamento de nove dimensões inerentes à prototipagem, caracterizando-a de maneira holística, sem desconsiderar as particularidades de cada disciplina. Os resultados deste estudo oferecem uma compreensão ampliada do tema e novas possibilidades em suas aplicações.

Palavras-chave: gestão da inovação; gestão de desenvolvimento de produtos; protótipo; prototipagem.

Área: ferramentas e métodos de desenvolvimento de produtos e serviços.

\section{INTRODUÇÃO}

A prototipagem é um conceito recorrente na Gestão de Desenvolvimento de Produtos (GDP) e tem fortes implicações na dinâmica de desenvolvimento de inovações em suas esferas técnica e gerencial. Nesse contexto, a prototipagem pode ser entendida como um ciclo onde o entendimento corrente de um projeto de produto é reunido e incorporado em uma representação (protótipo), culminando na realização de testes para determinar se refinamentos são necessários (CLARK; WHEELWRIGHT, 1993). Seu propósito é de reduzir incertezas, gerar aprendizado, solucionar problemas, integrar equipes, comunicar interna e externamente e gerenciar o cronograma de desenvolvimento (CLARK; WHEELWRIGHT, 1993; OTTO; WOOD, 2001; ULRICH; EPPINGER, 2012).

Por sua importância central para o debate da GDP, a prototipagem encontra em Clark e Wheelwright (1993) uma de suas discussões clássicas, na qual os autores a abordam sob um escopo gerencial e discutem as principais dimensões de duas filosofias: a tradicional, na qual a responsabilidade pelo protótipo avança de designers para engenheiros de manufatura na medida em que o projeto amadurece, e a periódica, na qual um time multifuncional é 
responsável por todos os ciclos primários de prototipagem. À época da publicação, diversas tecnologias para obtenção rápida de protótipos físicos já vinham ocupando espaço no debate da prototipagem. Otto e Wood (2001) confirmam isso, ao discutir não só os objetivos da prototipagem, mas também ao apresentar uma ampla análise atualizada do estado da arte dessas tecnologias.

Não obstante, os anos 2000 marcam a consolidação de uma série de perspectivas sobre o fenômeno da inovação que extrapolaram o recorte da GDP, trazendo várias abordagens para o papel e uso da prototipagem, muito embora o próprio termo "protótipo" tenha cedido lugar a outras terminologias em algumas destas disciplinas. Este estudo identifica usos do conceito de prototipagem, além da área de GDP, nas Tecnologias de Manufatura Rápida (TMR), na Engenharia de Software (ES), no Desenvolvimento de Serviços (DS), no Desenvolvimento de Empresas de Base Tecnológica (DEBT), no Design Thinking (DT) e no Movimento Maker (MM). Apesar das ricas possibilidades trazidas pelas novas disciplinas relacionadas à inovação para se discutir prototipagem, há uma lacuna no que diz respeito a uma abordagem transversal da prototipagem em si - como o conceito evolui numa perspectiva multidisciplinar e como diferentes disciplinas compartilham - ou poderiam compartilhar - o potencial do conceito. Dessa forma, o objetivo do presente trabalho é uma caracterização holística da prototipagem, resultante da identificação de suas principais dimensões.

A próxima seção apresenta a metodologia de pesquisa adotada, seguida por duas seções de revisão bibliográfica, a primeira discutindo as principais contribuições dos textos englobados pela GDP e a seguinte abordando as demais áreas. As dimensões da prototipagem são apresentadas na seção 5., seguida das considerações finais.

\section{METODOLOGIA DE PESQUISA}

A metodologia de pesquisa adotada é dividida em duas estratégias complementares: revisão bibliográfica sistemática e entrevistas semiestruturadas com especialistas.

\subsection{Revisão bibliográfica sistemática}

Foram listadas, a priori, cinco temáticas de estudo relacionáveis à inovação e que abordam a dinâmica de prototipagem: a Gestão de Desenvolvimento de Produtos, as Tecnologias de Manufatura Rápida, o Desenvolvimento de Serviços, o Desenvolvimento de Empresas de Base Tecnológica e o Movimento Maker. Ao longo da revisão, surgiram o Design Thinking e a Engenharia de Software.

A revisão bibliográfica se deu em sete ciclos de buscas, cada um com objetivos e escopos distintos, sumarizados no Quadro 1. Todas as buscas nas bases de dados indexadas adotaram "tópicos" como o critério de busca e foram ordenadas da mais recente a mais antiga, exceto nos casos explicitados. Dois critérios foram utilizados na seleção dos trabalhos: priorização de textos em que a prototipagem era o objeto de pesquisa e a representatividade do trabalho em relação à sua disciplina.

Convém destacar que a identificação das disciplinas se deu com base em dois critérios: pelo referencial teórico dos textos (uso significativo de citações de algum autor considerado seminal da disciplina) e pelo contexto de aplicação da prototipagem. Ainda que o fator de impacto tenha sido analisado, o periódico não foi considerado para a validação das disciplinas.

Para a GDP, o DEBT, o DT e a ES, obras amplamente reconhecidas como inaugurais das discussões foram consultadas como ponto de partida. Além disso, o software Mendeley indicou 23 textos, dos quais 7 foram selecionados. O Quadro 1 fornece mais informações. 
Por fim, é necessário ressaltar que o presente texto foi elaborado a partir de uma síntese da convergência das publicações exibidas nessa subseção 2.1., sendo que apenas uma parcela delas é apresentada e referenciada ao longo do trabalho.

Quadro 1. Revisão Bibliográfica Sistemática

\begin{tabular}{|c|c|c|c|c|c|c|}
\hline Ciclo & $\begin{array}{l}\text { Período da } \\
\text { Busca }\end{array}$ & Base & $\begin{array}{l}\text { Palavras-chave } \\
\text { utilizadas }\end{array}$ & $\begin{array}{l}\text { Registros } \\
\text { Retornados }\end{array}$ & Observações & Trabalhos Selecionados \\
\hline 1 & $\begin{array}{c}\text { Novembro } \\
\text { Dezembro } \\
16\end{array}$ & $\begin{array}{l}\text { Web of } \\
\text { Science }\end{array}$ & prototyp* & 229.445 & $\begin{array}{l}\text { Muitos desses trabalhos debatem a construção de um protótipo } \\
\text { específico ou utilizam o termo protótipo com um significado fora } \\
\text { do escopo da pesquisa }\end{array}$ & 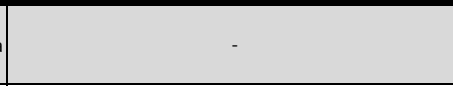 \\
\hline 2 & $\begin{array}{c}\text { Novembro } \\
\text { Dezembro } \\
16\end{array}$ & $\begin{array}{l}\text { Web of } \\
\text { Science }\end{array}$ & prototyp* & 401 & Foram buscados textos do tipo "review", com filtros & $\begin{array}{l}\text { TMR: Conley e Marcus (1997), Zorriassatine et al. } \\
\text { (2003), Cheah et al. (2005) }\end{array}$ \\
\hline 3 & $\begin{array}{c}\text { Novembro } \\
\text { Dezembro } \\
16\end{array}$ & $\begin{array}{l}\text { Web of } \\
\text { Science }\end{array}$ & $\begin{array}{l}\text { prototyp*AND } \\
\text { business }\end{array}$ & 570 & $\begin{array}{l}\text { Bicen e Johnson (2015) indicaram o DT ao citar a relação dessa } \\
\text { metodologia com a prototipagem. } \\
\text { Elverum e Welo (2016) deram indícios da ES por conta das } \\
\text { referências utilizadas }\end{array}$ & $\begin{array}{l}\text { GDP: Bicen e Johnson (2015), Elverum e Welo (2016). } \\
\text { Outros: Rayna e Striukova (2016) }\end{array}$ \\
\hline 4 & $\begin{array}{l}\text { Dezembro } \\
\text { Janeiro } 17\end{array}$ & $\begin{array}{l}\text { Web of } \\
\text { Science }\end{array}$ & $\begin{array}{l}\text { prototyp*AND } \\
\text { "product } \\
\text { development" }\end{array}$ & 1.357 & \begin{tabular}{|l|} 
Essa foi a busca de onde mais artigos foram selecionados, 11 no \\
total. \\
Thomke (1998) deu indícios da ES devido aos cases adotados. \\
Busca ordenada por relevância, depois por data de publicação \\
mais recente, depois por data de publicação mais antiga
\end{tabular} & $\begin{array}{l}\text { GDP: Thomke (1998), Veryzer (1998), Bullinger } \\
\text { (2000), Zhang et al. (2009), Thonke e Bell (2001), } \\
\text { Bernard e Fischer (2012). TMR: Kochan et al. (1999), } \\
\text { Onuh e Yusuf (1999), Hague et al. (2003), Choi e Chan } \\
\text { (2004). Outros: D'Adderio (2001) }\end{array}$ \\
\hline & $\begin{array}{l}\text { Dezembro- } \\
\text { Janeiro } 17\end{array}$ & $\begin{array}{l}\text { Web of } \\
\text { Science }\end{array}$ & \begin{tabular}{|c|} 
"minimum viable \\
product"
\end{tabular} & 8 & $\begin{array}{l}\text { Devido ao pequeno volume de resultados, outras bases de } \\
\text { dados foram utilizadas, gerando as buscas } 8 \text { e } 9\end{array}$ & - \\
\hline 5 & & Scopus & $\begin{array}{c}\text { "minimum viable } \\
\text { product" }\end{array}$ & 22 & - & DEBT: Yli-Huumo et al. (2015) \\
\hline & Janeiro 17 & $\begin{array}{c}\text { Science } \\
\text { direct }\end{array}$ & $\begin{array}{c}\text { "minimum viable } \\
\text { product" }\end{array}$ & 66 & - & DEBT: Duc e Abrahamsson (2016) \\
\hline 6 & $\begin{array}{c}\text { Fevereiro } \\
17\end{array}$ & $\begin{array}{l}\text { Web of } \\
\text { Science }\end{array}$ & \begin{tabular}{|c|} 
"service \\
prototyp*"
\end{tabular} & 85 & - & DS: Blomkvist (2016) \\
\hline 7 & Abril 17 & $\begin{array}{l}\text { Web of } \\
\text { Science }\end{array}$ & makerspaces & 55 & Ordenado por citações & $\begin{array}{l}\text { MM: Gershenfeld (2012), Sheridan et al. (2014), } \\
\text { Bosqué (2015) }\end{array}$ \\
\hline \multicolumn{7}{|c|}{ Artigos e livros identificados fora dos ciclos de buscas } \\
\hline \multirow{2}{*}{\multicolumn{3}{|c|}{$\begin{array}{l}\text { Livros } \\
\text { Mendeley }\end{array}$}} & \multirow{2}{*}{\multicolumn{4}{|c|}{$\begin{array}{c}\text { GDP: Clark e Wheelwright (1993), Otto e Wood (2001), Ulrich e Eppinger (2012). ES: Sommerville (2010). DETB: Ries (2012). DT: Brown (2010) } \\
\text { GDP: Houde e Hill (1997). TMR: Huang et al. (2015). ES e GDP. Gartzen et al. (2016). ES: Lim et al. (2008) }\end{array}$}} \\
\hline & & & \multicolumn{3}{|c|}{ GDP: Houde e Hill (1997). TMR: Huang et al. (2015). ES e GDP: Gartzen et al. (2016). ES: Lim et al. (2008) } & \\
\hline \multicolumn{3}{|c|}{ Referências de outros trabalhos } & \multicolumn{4}{|c|}{$\begin{array}{l}\text { GDP: Schrage (1993), Clark e Wheelwright (1994), Thomke e Fujimoto (2000). ES: Floyd (1984). DS: Voss (1992). DT: Brown (2008). DEBT / ES: Junk } \\
\text { (2000). MM: Dougherty (2012). Outros: Gerber e Carroll (2012) }\end{array}$} \\
\hline
\end{tabular}

Fonte: dos autores

\subsection{Entrevistas com especialistas}

As entrevistas foram desenvolvidas com base nas informações e questionamentos resultantes da revisão, tendo-se três objetivos em pauta: (i) reforçar e/ou questionar aspectos e padrões identificados na revisão de literatura; (ii) complementar as informações obtidas na literatura; (iii) validar hipóteses para a caracterização holística da prototipagem. Sete entrevistados foram selecionados, um para cada disciplina, apresentados no Quadro 2.

Quadro 2. Entrevistados e Forma de Entrevista

\begin{tabular}{|c|c|c|}
\hline Disciplina e Entrevistado & Qualificação & Forma de Entrevista \\
\hline \begin{tabular}{|l|} 
Gestão de Desenvolvimento de \\
Produtos - GDP1
\end{tabular} & $\begin{array}{l}\text { Mestre em Administração pela Universidade Federal de Minas Gerais (UFMG), com ênfase em Gestão da Inovação. } \\
\text { Gerenciou por três anos o portfólio de inovações de empresa de grande porte do segmento de energia }\end{array}$ & $\begin{array}{l}\text { Entrevista individual e presencial, gravada e } \\
\text { com duração de } 48 \text { minutos }\end{array}$ \\
\hline $\begin{array}{l}\text { Tecnologias de Manufatura } \\
\text { Rápida - TMR1 }\end{array}$ & $\begin{array}{l}\text { Doutor em Engenharia Metalúrgica pela Leeds University, Reino Unido. Possui oito anos de experiência na indústria, } \\
\text { desenvolvendo produtos e materiais. Leciona disciplinas de materiais e processos na University of Warwick, onde é o } \\
\text { Diretor do comitê de Educação Avançada em Tecnologia do Warwick Manufacturing Group }\end{array}$ & $\begin{array}{l}\text { Entrevista individual e virtual, gravada e com } \\
\text { duração de } 33 \text { minutos }\end{array}$ \\
\hline Engenharia de Software - ES1 & $\begin{array}{l}\text { Doutor em Ciência da Computação pela University of California, EUA . É professor do Departamento de Ciência da } \\
\text { Computação da UFMG e atua principalmente com o Desenvolvimento e Modelagem de Software }\end{array}$ & $\begin{array}{l}\text { Entrevista individual e presencial, gravada e } \\
\text { com duração de } 1 \text { he } 36 \text { minutos }\end{array}$ \\
\hline Design Thinking - DT1 & $\begin{array}{l}\text { Engenheiro de Produção pela Universidade Federal de Minas Gerais, empreendedor e sócio-diretor de empresa de } \\
\text { consultoria em gestão e inovação. Lecionou Workshops de Design Thinking }\end{array}$ & $\begin{array}{l}\text { Entrevista individual e presencial, gravada e } \\
\text { com duração de } 60 \text { minutos }\end{array}$ \\
\hline Design Thinking - DT2 & Designer pela Universidade Federal de Minas Gerais. Membro de um laboratório integrado de design na mesma universidade & Questionário enviado por e-mail \\
\hline \begin{tabular}{|l|} 
Desenvolvimento de Empresas \\
de Base Tecnológica - EBT1
\end{tabular} & $\begin{array}{l}\text { Engenheiro de Produção pela Universidade Federal de Minas Gerais. Atua há } 2 \text { anos como gestor em uma empresa onde } \\
\text { desenvolve trabalhos nas áreas de criação de negócios, desenvolvimento de produtos e gestão da inovação }\end{array}$ & $\begin{array}{l}\text { Entrevista individual e virtual, gravada e com } \\
\text { duração de } 1 \text { hora e } 32 \text { minutos }\end{array}$ \\
\hline Design de Serviços - DS1 & $\begin{array}{l}\text { Doutor em Engenharia de Produção pela Universidade de São Paulo. É professor do Departamento de Ciências } \\
\text { Administrativas. Parte de suas pesquisas são voltadas para modelos, métodos e técnicas para o desenvolvimento de serviços }\end{array}$ & $\begin{array}{l}\text { Entrevista individual e presencial, gravada e } \\
\text { com duração de } 24 \text { minutos }\end{array}$ \\
\hline Movimento Maker - MM1 & Doutor em Física pelo Centro Brasileiro de Pesquisas Físicas. É o responsável por um makerspace em Belo Horizonte & $\begin{array}{l}\text { Entrevista individual e presencial, gravada e } \\
\text { com duração de } 44 \text { minutos }\end{array}$ \\
\hline
\end{tabular}

\section{Fonte: dos autores}

A área profissional e/ou acadêmica na disciplina foi o critério de seleção. Para a execução das entrevistas, um roteiro semiestruturado foi elaborado, abordando os conceitos, os objetivos, as 
melhores práticas, as estratégias, os fatores culturais e organizacionais associados, as etapas de desenvolvimento envolvidas e as tendências para a prototipagem.

\section{GESTÃO DE DESENVOLVIMENTO DE PROTUDOS E A PROTOTIPAGEM}

\subsection{Uma abordagem gerencial da prototipagem na GDP e a cultura da prototipagem}

Clark e Wheelwright (1993) apresentam uma contribuição central à GDP e em especial à prototipagem. Os autores são categóricos ao afirmar que a sequência de ciclos de prototipagem forma a espinha dorsal do processo de desenvolvimento, sendo responsável não só pelo progresso técnico do projeto, mas também gerencial, agindo na resolução de problemas, na convergência de soluções, na comunicação, na integração de equipes e na estabilização e monitoramento do cronograma desenvolvimento (CLARK; WHEELWRIGHT, 1993).

Nesse contexto, os autores apresentam três filosofias de prototipagem: a tradicional, na qual as fases iniciais de design são responsabilidade de designers e as finais de engenheiros de manufatura; a tradicional revista, semelhante à tradicional, mas com maior envolvimento de consumidores e forte ênfase comercial; e a periódica, na qual um time multifuncional é responsável por todos os ciclos primários de prototipagem e pelo conjunto de testes de todos os aspectos do design em cada ciclo. O Quadro 3 apresenta uma análise comparativa dessas abordagens em termos das dimensões definidas por Clark e Wheelwright (1993).

Quadro 3. Filosofias de Prototipagem

\begin{tabular}{|c|c|c|c|}
\hline \multirow{2}{*}{ Dimensões } & \multicolumn{3}{|c|}{ Modelos } \\
\hline & Tradicional & Tradicional Revisado & Periódico \\
\hline Força Motriz & Performance Técnica & Performance Técnica/Comercial & $\begin{array}{l}\text { Performance do sistema / } \\
\text { integração interfuncional }\end{array}$ \\
\hline Foco & Avaliar o Design & $\begin{array}{l}\text { Avaliar o Design / Satisfação do } \\
\text { Cliente }\end{array}$ & Solução superior do sistema \\
\hline \multicolumn{4}{|l|}{ Controle de Ciclos } \\
\hline Inicial & Engenharia & Engenharia & Equipe de peso (plataforma) \\
\hline Médio & Engenharia & Engenharia & Equipe de peso (plataforma) \\
\hline Posterior & Manufatura & Manufatura & Equipe de peso (plataforma) \\
\hline \multicolumn{4}{|c|}{ Responsabilidade pela Construção } \\
\hline Inicial & Subcontratada & Setor de protótipos da engenharia & Setor de protótipos da engenharia \\
\hline Médio & Setor de protótipos da engenharia & Setor de protótipos da engenharia & Linha de produção/manufatura \\
\hline Posterior & Fábrica & Fábrica & Linha de produção comercial \\
\hline Papel/envolvimento do cliente & Limitado às últimas fases de testes & $\begin{array}{l}\text { Fase inicial: evolução dos "mock-up" } \\
\text { Fase posterior: evolução do sistema }\end{array}$ & \begin{tabular}{|l} 
Testes de protótipos \\
(confiabilidade) junto ao Cliente
\end{tabular} \\
\hline Critérios de testes & $\begin{array}{l}\text { Funcionalidade do componente / do } \\
\text { sistema }\end{array}$ & $\begin{array}{l}\text { Funcionalidade / confiabilidade do } \\
\text { componente e sistema }\end{array}$ & $\begin{array}{l}\text { Funcionalidade do sistema para } \\
\text { produto e processo }\end{array}$ \\
\hline $\begin{array}{l}\text { Ligação entre marcos do } \\
\text { gerenciamento }\end{array}$ & $\begin{array}{l}\text { Limitado: pontos de controle e } \\
\text { revisão conforme programa }\end{array}$ & $\begin{array}{l}\text { Fase inicial: evolução dos "mock-up" } \\
\text { Fase posterior: evolução do sistema }\end{array}$ & $\begin{array}{l}\text { Ciclos de protótipos são os pontos } \\
\text { de gerenciamento }\end{array}$ \\
\hline
\end{tabular}

Fonte: adaptado de Clark e Wheelwright (1993)

Os objetivos da prototipagem sugeridos por Otto e Wood (2001) e por Ulrich e Eppinger (2012) são semelhantes aos propostos por Clark e Wheelwright (1993). Entretanto, esses autores orientam seus debates a decisões técnicas de engenharia que envolvem a prototipagem, como por exemplo o meio e o material do protótipo.

Schrage (1993) debate a relação entre a cultura organizacional e a prototipagem, denominada de cultura de prototipagem. Alguns dos fatores constituintes dessa cultura são: a gestão do diálogo entre especificações e protótipos, a dimensão política inerente a esses artefatos e a disputa entre uma cultura orientada a especificações e outra orientada a protótipos (SCHRAGE, 1993). A Figura 1 apresenta o modelo proposto pelo autor para diagnosticar, independente do contexto, a cultura de prototipagem de uma organização. 
Figura 1. Matriz de Diagnóstico da Cultura de Prototipagem
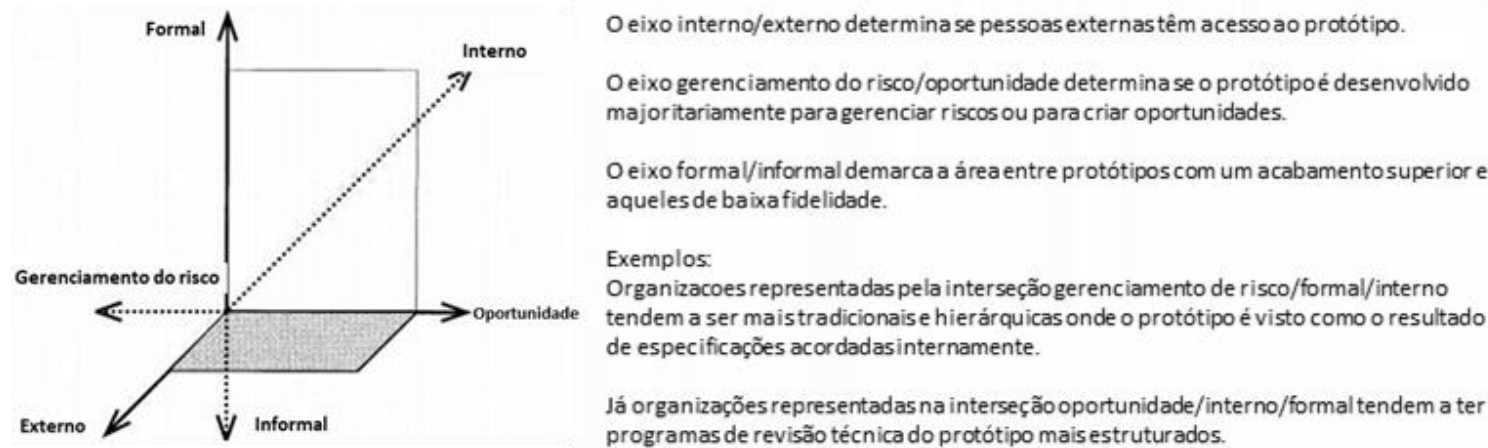

Fonte: adaptado de Schrage (1993)

\subsection{Operacionalizando a prototipagem e melhores práticas}

Clark e Wheelwright (1994) e Thomke (1998) discutem a implementação da prototipagem por meio de ciclos virtuosos de design-construção-teste-análise. Nesse contexto, os dois primeiros autores debatem a relação entre esses ciclos e as formas de comunicação e integração de equipes de design. Já o segundo autor debate a alternância ótima entre modos de experimentação de forma a reduzir os custos e o leadtime de desenvolvimento.

A literatura identifica três categorias nas quais a prototipagem pode gerar valor no processo de desenvolvimento: o artefato (protótipo), o processo de criação do artefato (prototipagem) e o experimento feito com o artefato. (ELVERUM; WELO, 2016).

Há também decisões e estratégias que envolvem a prototipagem e que devem ser consideradas ao longo do desenvolvimento. Thomke e Bell (2001), por exemplo, sugerem um modelo para otimizar o número de testes realizados em um projeto. Christie et al. (2012) definem uma estratégia de prototipagem, composta por fatores que devem ser debatidos a cada ciclo de prototipagem.

Por fim, há textos que debatem a relação entre modelos alternativos de desenvolvimento e a prototipagem. Por exemplo, Veryzer (1998) discute o papel da prototipagem em modelos de desenvolvimento descontínuos que se desenrolam desde a tecnologia até o produto final.

\section{PROTOTIPAGEM: PERSPECTIVAS DISCIPLINARES}

Essa seção apresenta a perspectiva das demais disciplinas que abordam a prototipagem, respeitando a seguinte ordem: TMR, ES, DEBT, DT, DS, e MM.

As publicações envolvidas pelas Tecnologias de Manufatura Rápida são focadas, como o próprio nome já indica, nas tecnologias de construção dos protótipos, dando menor ênfase ao desenvolvimento de produtos e a aspectos gerenciais. Onuh e Yusuf (1999), por exemplo, são exceção ao apresentar uma análise das principais aplicações dessas tecnologias para o desenvolvimento rápido de produtos. Conley e Marcus (1997), por outro lado, apresentam uma revisão do estado da arte de práticas comerciais e de pesquisa, em especial as tecnologias de fabricação de forma sólida livre. Publicações mais recentes debatem o uso de tecnologias originalmente de prototipagem rápida para a manufatura direta de componentes e produtos.

Já na Engenharia de Software, Sommerville (2010) discute a prototipagem como um método para reduzir os custos de retrabalho de um projeto de desenvolvimento em se deseja evitar mudanças (SOMMERVILLE, 2010). Floyd (1984), por outro lado, defende que a prototipagem no contexto da ES é um mecanismo de aprendizado. Diferente da manufatura que, segundo a autora, entende o protótipo como sendo um primeiro "tipo" do produto, a 
prototipagem na ES se refere a uma fase específica do desenvolvimento, na qual um modelo é produzido antecipadamente para testar e direcionar os esforços.

No que concerne o Empreendedorismo de Base Tecnológica, Ries (2012) define que os ciclos de construir-avaliar-aprender estão no cerne do modelo da startup enxuta. A operacionalização desses ciclos são os Produtos Mínimos Viáveis (MVPs), que consistem na versão do produto que permite uma volta completa no ciclo, com o mínimo esforço e o menor tempo de desenvolvimento (RIES, 2012). O autor diferencia esse conceito da prototipagem, uma vez que o protótipo é projetado para responder a perguntas técnicas do produto, enquanto o MVP busca testar hipóteses fundamentais do negócio. É possível identificar na literatura convergências e divergências em relação à distinção proposta por Ries (2012). Por exemplo, Yli-Huumo et al. (2015) entendem que o MVP é um protótipo simples do produto, anexável ao modelo de negócios da empresa. Nessa perspectiva, um mesmo artefato é utilizado para mensurar e avaliar tanto as especificações do produto quanto do modelo de negócios.

No Design Thinking, Brown (2010) entende a prototipagem como a disposição de um indivíduo testar alguma hipótese construindo um objeto. Mais do que em outros contextos, no DT a prototipagem assume o papel de um mecanismo criativo. $\mathrm{O}$ autor defende, por exemplo, que muitos protótipos rudimentares de baixo custo e de rápida construção devem ser realizados, especialmente nas fases iniciais de desenvolvimento. Assim sendo, protótipos só devem consumir os recursos necessários para gerar avaliações e melhorias. Além disso, Brown (2010) ainda defende que a prototipagem pode ser aplicada ao desenvolvimento de estratégias de negócios (envolvendo diferentes agentes na sua construção e desdobramento) e ao desenvolvimento organizacional (induzindo a mudança e adaptação necessária).

No Design de Serviços, Voss (1992) adota a prototipagem como mecanismo central de seu modelo para inovações conceituais de serviços. Em um estudo mais atualizado, Blomkvist (2016) defende que a prototipagem é utilizada no desenvolvimento de serviços para aprimorar seu design, explorar formas de implementa-lo e auxiliar na tomada de decisão (BLOMKVIST, 2016). O autor define quatro níveis em que a prototipagem gera valor ao DS: o artefato, o uso do artefato, o contexto da interação e o serviço, que incorpora os três níveis.

Em uma pesquisa que buscou entender as características que garantem o aprendizado em makerspaces, parte constituinte do Movimento Maker, Sheridan et al. (2014) chegam à conclusão de que o aprendizado nestes ambientes está profundamente enraizado na experiência do 'fazer'. Tecnologias de prototipagem, em especial a prototipagem rápida, permitem aos participantes 'fazer' objetos de maneira criativa, seja na concepção ou aperfeiçoando ideias (SHERIDAN et al., 2014).

\section{DISCUSSÃO}

A seção 3 deste trabalho apresenta algumas das dimensões da prototipagem que são discutidas no contexto da GDP, atualizando e iluminando o corpo de conhecimento que envolve essa abordagem. Entretanto, não há consenso em relação às definições e ao alcance da abordagem (técnico, gerencial ou ambos). Quando se expande a análise para as disciplinas apresentadas na seção subsequente, novas perspectivas e entendimentos trazem ainda maior variabilidade (e possibilidades mais amplas) para essas questões.

Deve-se esclarecer que a falta de consenso entre os autores não resulta, até onde se sabe, em uma disputa entre diferentes entendimentos. O que se verifica, por outro lado, é a subjetividade das dimensões da prototipagem, variando de maneira significativa entre os autores e resultando, em alguns casos, na limitação do diálogo entre diferentes abordagens. 
Dada a ausência de publicações que busquem analisar a prototipagem de maneira holística e que mapeiem suas diferentes dimensões, essa seção almeja este fim.

\subsection{Dimensões da Prototipagem}

A análise dos textos e das entrevistas permitiu a identificação de algumas das dimensões constituintes e inerentes à prototipagem, sendo elas independentes das abordagens e dos contextos de aplicação. Serviram como direcionadores principais desse levantamento os trabalhos de Clark e Wheelwright (1993) e de Schrage (1993).

- Objeto da prototipagem.

O que é passível de ser prototipado. A literatura e entrevistados da GDP, da TMR, da ES e do Movimento Maker indicaram que seus objetos se relacionam aos respectivos produtos de cada área e, em alguns casos, aos processos de fabricação destes. No DEBT, Ries (2012) escreve que protótipos são utilizados para o desenvolvimento de produtos, enquanto o entrevistado indicou o seu uso para produtos, serviços e modelos de negócios. Por fim, a literatura do Design Thinking adota a ideia de protótipo para produtos, processos, serviços, modelos de negócios e estratégias empresariais. $\mathrm{O}$ entrevistado dessa disciplina considera o conceito apenas para produtos (tangíveis e intangíveis).

\section{- Conceito de protótipo.}

Quais os aspectos, se houver algum deles, que necessariamente caracterizam a representação do objeto da prototipagem. Em geral, esses são frutos de uma série de decisões de engenharia. Por exemplo, na literatura da GDP e da TMR, há um debate interno que busca definir o que é um protótipo em termos dos atributos da representação do objeto. Por exemplo, Otto e Wood (2001) definem que apenas artefatos físicos podem ser considerados protótipos, enquanto Clark e Wheelwright (1993) não fazem essa distinção.

- Força motriz da prototipagem.

É o objetivo final da prototipagem e pode ser um fator crítico para diferenciar abordagens técnicas de abordagens gerenciais. Clark e Wheelwright (1993) já previram quatro delas: performance técnica, performance comercial, performance do sistema e integração interfuncional. Entretanto, há ainda outros direcionadores. Schrage (1993), por exemplo, debate o que pode ser considerado como uma força motriz política. Já Brown (2010) apresenta forte ênfase na exploração criativa para posterior desenvolvimento técnico. Por fim, verifica-se uma força motriz para angariar investimentos em um projeto.

- A prototipagem como um mecanismo para a convergência ou divergência de soluções.

Schrage (1993) introduz essa dimensão, discutindo que protótipos podem ser utilizados para gerenciar os riscos de desenvolvimento ou para criar novas oportunidades. Pode-se traduzir essa dimensão em termos do duelo entre convergência (redução de riscos, soluções ineficientes, etc.) e divergência (levantamento de mais possibilidades, ideias e perspectivas) de soluções. Essa dimensão impacta diferentes decisões de prototipagem, como por exemplo a quantidade de conceitos de protótipos que serão desenvolvidos para uma solução. Deve-se ressaltar que há uma tendência na literatura e entre os entrevistados para o uso da prototipagem majoritariamente com foco convergente, mas também deve ser considerado que muitos fatores contingenciais como o custo de desenvolvimento e os riscos envolvidos afetam diretamente esta dimensão.

- Envolvimento de agentes externos. 
Define o grau no qual agentes externos às equipes de desenvolvimento, estão envolvidos na prototipagem. Há casos em que não há envolvimento algum, outros em que o envolvimento é limitado a uma parte do desenvolvimento e outros em que o engajamento é essencial, com o agente se envolvendo diretamente no desenvolvimento. Sua importância foi ressaltada por DEBT1, que sugeriu como uma tendência para a prototipagem a crescente valorização da competência de engajar terceiros e a correta interpretação dessas avaliações.

\section{- Conceito da prototipagem como um ciclo.}

Existem duas formas distintas em se conceituar o que é a prototipagem. De um lado, tem-se a prototipagem como um processo que envolve etapas de design, construção, teste e melhoria. Do outro, a prototipagem compreende apenas a construção do artefato. Esses extremos podem ter um impacto no resultado da abordagem adotada e certamente alteram a nomenclatura e conceitos utilizados. Por exemplo, Thomke (1998) adota a terminologia de "ciclos de aprendizado na experimentação" para se referir às etapas de design, construção, execução e análise de um artefato, que pode ser um protótipo ou não.

- Frequência, fidelidade e estratégia dos ciclos.

Uma questão relevante no contexto da prototipagem é a frequência na qual os ciclos de prototipagem são executados, considerando-se a fidelidade incorporada ao protótipo e a forma na qual os ciclos se relacionam. Essas são decisões de condução da prototipagem, o que é designado na literatura como estratégia de prototipagem. De uma maneira geral, é possível alterar os parâmetros atribuídos à frequência (um único teste ou múltiplos testes), fidelidade (total, na qual objetiva-se a fiel replicação no protótipo daquilo que está sendo desenvolvido, ou variável, na qual se simplifica algo na representação) e estratégia (testes totalmente sobrepostos, parcialmente sobrepostos ou complementares) entre um projeto de desenvolvimento e outro.

\section{- Etapa de Desenvolvimento.}

Em qual etapa de desenvolvimento a prototipagem é aplicada. De uma maneira geral, verificase que a grande maioria dos autores entende que a prototipagem pode ser aplicada a qualquer etapa, independente do objeto adotado. Essa dimensão, entretanto, é uma das que mais depende do contexto de aplicação, visto que diferentes modelos de desenvolvimento são adotados, cada um deles com suas particularidades.

\section{- Organização das equipes de desenvolvimento.}

Diz respeito a como as equipes de desenvolvimento são organizadas e, em especial, como pessoas com diferentes funções e bases de conhecimento são envolvidas ou não no desenvolvimento. Apesar de poucos textos tratarem diretamente da importância da organização das equipes de desenvolvimento, Clark e Wheelwright (1993) depositam grande importância nessa dimensão, debatendo a prototipagem como um mecanismo que viabiliza a integração interfuncional. Nesse sentido, o protótipo é um objeto de fronteira onde diferentes agentes podem interagir, utilizando uma linguagem comum. Também é argumentado na literatura que o protótipo age no sentido de normalizar o entendimento a respeito do projeto, reduzindo assim incertezas e falhas de comunicação.

As nove dimensões da prototipagem, por fim, permitem que se faça uma análise comparativa de diferentes abordagens, considerando as particularidades de cada uma delas dentro de uma estrutura comum, reintegrando o conceito e permitindo um olhar holístico sobre o tema. $\mathrm{O}$ Quadro 4 apresenta um primeiro esboço dessa análise. Nele, a abordagem de alguns autores/entrevistados da GDP, TMR, DEBT e DT são classificadas em termos das dimensões. 
Células em branco indicam informações ausentes ou insuficientes. Além disso, as dimensões "etapa de desenvolvimento", "organização das equipes de desenvolvimento" e "a frequência, fidelidade e estratégia dos ciclos" foram omitidas devido à falta de informação.

Quadro 4. Estrutura comparativa de abordagens

\begin{tabular}{|c|c|c|c|c|c|c|c|c|}
\hline \multirow[b]{2}{*}{ Disciplina } & \multirow[b]{2}{*}{$\begin{array}{l}\text { Autores / } \\
\text { Entrevistados }\end{array}$} & \multirow[b]{2}{*}{ Observaçōes } & \multicolumn{6}{|c|}{ Dimensões da Prototipagem } \\
\hline & & & $\begin{array}{l}\text { Objeto da } \\
\text { prototipagem }\end{array}$ & $\begin{array}{l}\text { Conceito de } \\
\text { protótipo }\end{array}$ & $\begin{array}{l}\text { Força motriz da } \\
\text { prototipagem }\end{array}$ & $\begin{array}{c}\text { Envolvimento de agentes } \\
\text { externos }\end{array}$ & $\begin{array}{c}\text { Mecanismo para a } \\
\text { convergência } X \\
\text { divergência de } \\
\text { soluções }\end{array}$ & $\begin{array}{l}\text { O conceito da prototipagem } \\
\text { como um ciclo }\end{array}$ \\
\hline TMR & TMR1 & - & $\begin{array}{c}\text { Produtos, processos e } \\
\text { serviços }\end{array}$ & \begin{tabular}{|c|} 
Apenas \\
representações fisicas \\
e protótipos virtuais
\end{tabular} & Performance técnica & - & Convergência & \begin{tabular}{|c|}
$\begin{array}{c}\text { Ciclo de design, construção, } \\
\text { teste e melhoria, mas com } \\
\text { maior ênfase à construção e } \\
\text { ao artefato }\end{array}$ \\
\end{tabular} \\
\hline \multirow[b]{2}{*}{ DEBT } & Ries (2012) & $\begin{array}{c}\text { Protótipo (MVP não } \\
\text { é considerado) }\end{array}$ & Produtos & $\begin{array}{c}\text { Não especifica } \\
\text { aspectos obrigatórios }\end{array}$ & Performance técnica & $\begin{array}{c}\text { Limitado às fases finais de } \\
\text { testes }\end{array}$ & Convergência & $\begin{array}{l}\text { Ciclo de design, construção, } \\
\text { teste e melhoria }\end{array}$ \\
\hline & DEBT1 & - & $\begin{array}{l}\text { Produtos, serviços e } \\
\text { modelos de negócios }\end{array}$ & $\begin{array}{c}\text { Não especifica } \\
\text { aspectos obrigatórios }\end{array}$ & $\begin{array}{l}\text { Pode ser técnica, } \\
\text { comercial, do } \\
\text { sistema, } \\
\text { demonstração para } \\
\text { investidores ou } \\
\text { exploração criativa } \\
\end{array}$ & \begin{tabular}{|c|} 
Stakeholders são \\
envolvidos em muitos \\
testes e podem direcionar \\
os esforços de \\
desenvolvimento
\end{tabular} & $\begin{array}{c}\text { Majoritariamente } \\
\text { convergência, mas } \\
\text { também há grande } \\
\text { espaço para } \\
\text { divergência }\end{array}$ & $\begin{array}{l}\text { Ciclo de design, construção, } \\
\text { teste e melhoria }\end{array}$ \\
\hline
\end{tabular}

Fonte: dos autores

\section{CONCLUSÃO}

Em 1993, Clark e Wheelwright publicaram seu livro seminal para a Gestão de Desenvolvimento de Produtos, no qual discutiam a importância da prototipagem como uma abordagem gerencial. Desde então, o conceito transbordou para diversas áreas do conhecimento afins à temática da inovação abrindo novas possibilidades ao tema, mas com poucos esforços de integração entre as diferentes perspectivas de análise. Esse estudo representa um esforço para tal integração, ao delinear as dimensões que caracterizam as abordagens de prototipagem.

Ainda que os resultados deste trabalho permitam o início de uma caracterização holística da prototipagem, ressalvas metodológicas devem ser feitas. A revisão bibliográfica parte da GDP e traz naturalmente consigo os vieses típicos desta perspectiva. As entrevistas, também, consideraram apenas um representante de cada área, além de ter um predomínio de uma visão de engenharia em termos da formação dos entrevistados.

Por fim, a definição das dimensões da prototipagem possui implicações imediatas para a dinâmica de inovações e da própria prototipagem, facilitando o intercâmbio de melhores práticas entre diferentes disciplinas. Dessa forma, Gerentes de Desenvolvimento podem alinhar estratégia empresarial, cultura organizacional e dimensões da prototipagem para ter resultados mais consistentes. Entretanto, para que seus impactos sejam amplificados, é necessário estudar em com maior profundidade o escopo de cada dimensão e avaliar se essas dimensões são representativas ou se há ainda outras a se considerar.

\section{REFERÊNCIAS}

BLOMKVIST, J. Benefits of service level prototyping. Design Journal, v. 19, n. 4, p. 545564, 2016.

BROWN, T. Design Thinking: uma metodologia poderosa para decretar o fim das velhas ideias. Primeira ed. Rio de Janeiro: Elsevier, 2010. 249 p. 
CHRISTIE, E. J. et al. Prototyping strategies: literature review and identification of critical variables. ASEE Annual Conference and Exposition, San Antonio, p. 1122-1154, 2012.

CLARK, K. B.; WHEELWRIGHT, S. C. Managing new product and process development: text and cases. Primeira ed. New York: The Free Press, 1993. 896 p.

CLARK, K. B.; WHEELWRIGHT, S. C. Accelerating the design- build- test cycle for effective product development. International Marketing Review, v. 11, n. 1, p. 32-46, 1994.

CONLEY, J. G.; MARCUS, H. L. Rapid prototyping and solid free form fabrication. Journal of Manufacturing Science and Engineering, v. 119, n. 4, p. 811-816, 1997.

ELVERUM, C. W.; WELO, T. Leveraging prototypes to generate value in the concept-toproduction process: a qualitative study of the automotive industry. International Journal of Production Research, v. 54, n. 10, p. 3006-3018, 2016.

FLOYD, C. A systematic look at prototyping. In: BUDDE, R.; KUHLENKAMP, K.; MATHIASSEN, L.; ZULLIGHOVEN, H. (Eds). Approaches to Prototyping. Berlin: Springer-Verlag, 1984. p. 1-18.

ONUH, S. O.; YUSUF, Y. Y. Rapid prototyping technology: applications and benefits for rapid product development. Journal of Intelligent Manufacturing, v. 10, n. 3, p. 301-311, 1999.

OTTO, K. N.; WOOD, K. L. Product design: techniques in reverse engineering and new product development. Primeira ed. Upper Saddle River: Prentice Hall, 2001. 1104 p.

RIES, E. A Startup Enxuta: como os empreendedores atuais utilizam a inovação contínua para criar empresas extremamente bem-sucedidas. Primeira ed. São Paulo: Lua de Papel, 2012. (Kindle).

SCHRAGE, M. The culture(s) of prototyping. Design Management Journal, v. 4, n. 1, p. 5565, 1993.

SHERIDAN, K. et al. Learning in the making: comparative case study of three makerspaces. Harvard Educational Review, v. 84, n. 4, p. 505-532, 2014.

SOMMERVILLE, I. Software engineering. Nona ed. Boston: Pearson, 2010. 792 p.

THOMKE, S.; BELL, D. E. Sequential Testing in Product Development. Management Science v. 47, n. 2, p. 308-323, 2001.

THOMKE, S. Managing experimentation in the design of new products. Management Science, v. 44, n. 6, p. 743-762, 1998.

ULRICH, K. T.; EPPINGER, S. D. Product design and development. Quinta ed. Nova Iorque: McGraw-Hil/Irwin, 2012. 415 p.

VERYZER, R. Discontinuous innovation and the new product development process. Journal of Product Innovation Management, v. 15, n. 4, p. 304-321, 1998.

VOSS, C. Measurement of innovation and design performance in services. Design Management Journal, v. 3, n. 1, p. 40-46, 1992.

YLI-HUUMO, J. et al. The relationship between business model experimentation and technical debt. In: INTERNATIONAL CONFERENCE OF SOFTWARE BUSINESS, 2015, Braga. Suiça: Springer, 2015. V. 210. p.17-29. 